\title{
Lidil
}

Revue de linguistique et de didactique des langues

$48 \mid 2013$

L'émotion et l'apprentissage des langues

\section{Foued Laroussi et Marie-Claude Penloup (dir.), Identités langagières, Mélanges offerts à Régine Delamotte}

Presses universitaires de Rouen et du Havre (PURH), 2013

\section{Virginie Lapique}

\section{OpenEdition}

Journals

Édition électronique

URL : http://journals.openedition.org/lidil/3343

DOI : 10.4000/lidil.3343

ISSN : $1960-6052$

Éditeur

UGA Éditions/Université Grenoble Alpes

Édition imprimée

Date de publication : 1 novembre 2013

Pagination : 215-217

ISBN : 978-2-84310-260-8

ISSN : $1146-6480$

\section{Référence électronique}

Virginie Lapique, « Foued Laroussi et Marie-Claude Penloup (dir.), Identités langagières, Mélanges offerts à Régine Delamotte ", Lidil [En ligne], 48 | 2013, mis en ligne le 01 mai 2015, consulté le 25 septembre 2020. URL : http://journals.openedition.org/lidil/3343 ; DOI : https://doi.org/10.4000/lidil.3343 
Foued Laroussi et Marie-Claude Penloup (dir.), Identités langagières, Mélanges offerts à Régine Delamotte, Presses universitaires de Rouen et du Havre (PURH), 2013

Comment rendre, surtout en quelques mots, le pluriel et le mouvement, sans figer ni réduire? C'est l'entreprise difficile qui nous attend pour présenter l'ouvrage d'hommages à Régine Delamotte, dont le titre, Identités langagières, au pluriel, et le sous-titre, qui met en avant l'hétérogénéité (Mélanges), dessinent les contours d'un horizon de lecture foisonnant, ouvert et stimulant. On croise au fil des pages le «cercle proche» de la chercheuse, autour de Frédéric François, qui a encadré sa thèse sur la pathologie du langage, soutenue en 1973, et les sociolinguistes Christiane et Jean-Baptiste Marcellesi, qui ont accompagné ses débuts à l'Université de Rouen. À l'implication des collègues rouennais(ses) du laboratoire DYSOLA, Dynamiques sociales et langagières, Foued Laroussi, Marie-Claude Penloup, qui coordonnent l'ouvrage, mais aussi Mehmet Ali Akinci, Évelyne Delabarre, Philippe Lane, Patrice Cohen, Sophie Devineau, s'ajoutent des contributions de chercheur(se)s, dont certain(e)s sont d'ancien(ne)s étudiant(e)s, comme Catherine Julien-Kamal, venu(e)s d'horizons variés. C'est toute la dynamique de la recherche en sciences du langage, dans sa dimension diachronique et spatiale, et dans la variété des champs concernés (linguistique, sociolinguistique, psycholinguistique, (socio)didactique des langues, etc.) qui est illustrée par la diversité des contributions réunies, de l'analyse par Christine Deprez des rapports qu'entretenaient deux reines de France, l'Autrichienne Marie-Antoinette et l'Italo-Allemande Marie de Médicis, avec la langue française, à l'exploration par Dora Carpenter du discours politique sur la société, les genres et les langues que tient le film La graine et le mulet de Kechiche : l'ouvrage est à l'image des choix scientifiques de la chercheuse, qui explore les sujets langagiers dans leur hétérogénéité, «qu'il s'agisse d'enfants dans un monde adulto-centré ou de locuteurs plurilingues dans une France imprégnée par un idéal monolingue» (Avant-lire, p. 8).

Trois contributions forment les portraits, premier mouvement du triptyque autour duquel est organisé l'ouvrage, et nous invitent à aller à la rencontre de Régine Delamotte, en mettant en regard sa personnalité, son œuvre, et le contexte d'une époque marquée par de nombreux combats sociaux et politiques, qui s'éclairent mutuellement, et en mettant au jour les enjeux éthiques et politiques qui en constituent le fil rouge, «car c'est bien dans l'éthique que tout son travail de chercheuse plonge 
ses racines » (Rispail, p. 23). Poser des questions, être mis(e) en mouvement par une insatiable curiosité des gens, les aimer, pratiquer le féminisme et la modestie, autant de lignes de vie et de travail, autant de piliers structurants de son œuvre.

Suivent vingt-quatre contributions, les hommages, qui se présentent comme autant de dialogues ouverts avec Régine Delamotte et auxquels le(la) lecteur(trice) est invité(e) à participer. Tout au plus pouvonsnous souligner la multiplicité des angles sous lesquels le rapport au langage est appréhendé, multiplicité gage de richesse, par les intersections, les complémentarités et les écarts qu'elle autorise. Au fil des pages, le lecteur (re)construit des connexions entre les contributions (les analyses de Francis Grossmann entrent en résonance avec celles qu'Elisabeth Bautier consacre aux difficultés scolaires des élèves, les développements d'Aliyah Morgenstern et Christophe Parisse prolongent l'étude de Mehmet Ali Akinci sur les narrations enfantines), et des ponts sont jetés avec les travaux de Régine Delamotte, dont ces contributions soulignent, à travers leurs jeux de miroirs, la «joyeuse hétérogénéité» (Avant-lire, p. 8). Ainsi, la thématique du plurilinguisme fait l'objet d'éclairages croisés, mêlant l'analyse des représentations d'enseignant(e)s d'un lycée professionnel de Grenoble face à l'hétérogénéité linguistique de leurs élèves (Jacqueline Billiez, Patricia Lambert), à la rencontre de la pluralité linguistique par des élèves de maternelle scolarisés dans des ZEP (Zones d'éducation prioritaires) françaises (Marie-Thérèse Vasseur), à l'étude de parcours langagiers d'étudiants mahorais (Évelyne Delabarre) ou égyptiens (Catherine Julien-Kamal), en passant par la recherche de pistes didactiques pour les classes bilingues vietnamiennes (Duc Su Pham). Derrière cette variété, qui rappelle la diversité des terrains d'investigation explorés par la chercheuse, les contributions puisent leur unité dans l'ouverture de Régine Delamotte aux autres en tant que personnes langagières, que ces autres soient différents par leur âge ou leurs usages linguistiques et culturels, et dans la volonté de placer l'hétérogénéité, «caractéristique intrinsèque des dispositions humaines » (Castellotti, p. 75), au cœur de la réflexion, alors même qu'elle est trop souvent, en éducation comme en (socio)linguistique, soit occultée, soit considérée comme un problème qu'il faudrait résoudre (Caitucoli).

Les témoignages, troisième volet de l'ouvrage (Jean-Baptiste et Christiane Marcellesi, Jacques Treignier, Alain Vom Hofe), mettent en lumière l'influence de Régine Delamotte dans la diffusion de la sociolinguistique, sa conviction que la recherche doit être impulsée par des équipes pluri-catégorielles et ouverte sur l'interdisciplinarité, et ne 
font que confirmer en quoi ces Mélanges, en croisant les regards sur le langage, en explorant des terrains sociolinguistiques variés (Tunisie, Mayotte, Égypte, Viêt Nam...), en ouvrant des fenêtres sur l'école (à la fois du côté des enseignant(e)s et des élèves), sans jamais perdre de vue l'engagement dans une démarche éthique, constituent une belle invitation à «penser avec» la chercheuse, à nous mettre en mouvement vers ses travaux, et vers les multiples pistes qu'ils ouvrent.

Virginie Lapique Doctorante au CELEC/CEDICLEC, St-Étienne

Régine Delamotte et Mehmet-Ali Akinci (dir.), Récits d'enfants. Développement, genre, contexte, Presses universitaires de Rouen et du Havre, 2012, 380 p.

«Enfin un ouvrage consacré aux récits d'enfants produits à l'oral!», pourrait-on dire, car comme l'annonce la quatrième de couverture, pour une fois, «récit» ne rime pas avec «écrit». Or, comme le notent les auteurs dans la présentation, le récit oral est présent partout : dans les histoires qu'on raconte en famille au jeune enfant, dans les moments de langage à l'école maternelle, lorsqu'on lui demande d'évoquer ce qu'il a vécu la veille, et plus tard dans la conversation courante, où l'on se raconte ce qu'on a entendu, vu ou vécu au quotidien.

L'ouvrage s'inscrit dans la lignée des travaux pionniers de Frédéric François et de son équipe sur le langage enfantin, et il en épouse les lignes de force : une grande ouverture analytique (absence d'un modèle dominant), un intérêt pour toutes les formes de réalisation narrative, et la mise en évidence de l'hétérogénéité des pratiques narratives enfantines. Qui dit «hétérogénéité» dit «variation». Or justement, autre originalité de l'entreprise : il s'agit moins de fournir au lecteur une vision de ce que serait LE récit enfantin, que de lui montrer LES récits d'enfants, dans toute la palette de leur diversité. Cette hétérogénéité des formes de narration, le lecteur est appelé à la découvrir dans les trois dimensions qui donnent sa structure à l'ouvrage : le développement langagier ( $1^{\mathrm{re}}$ partie), le genre narratif $\left(2^{\mathrm{e}}\right.$ partie $)$, les contextes de production ( $3^{\mathrm{e}}$ partie).

Ainsi, dans la première partie, le lecteur a d'abord l'occasion de découvrir, grâce à la contribution de Aliyah Morgenstern, le récit enfantin en émergence, à travers les verbalisations d'un jeune enfant de 